\title{
L'historiographie germanophone sur les lois barbares : centres de gravité, évolutions, desiderata
}

Thomas Lienhard

\section{(2) OpenEdition}

\section{Journals}

Édition électronique

URL : http://journals.openedition.org/ifha/243

DOI : $10.4000 /$ ifha. 243

ISSN : 2198-8943

\section{Éditeur}

IFRA - Institut franco-allemand (sciences historiques et sociales)

\section{Édition imprimée}

Date de publication : 1 janvier 2010

Pagination : 133-163

ISSN : 2190-0078

\section{Référence électronique}

Thomas Lienhard, «L'historiographie germanophone sur les lois barbares : centres de gravité

évolutions, desiderata », Revue de l'IFHA [En ligne], 2 | 2010, mis en ligne le 01 février 2013, consulté le 01 mai 2019. URL : http://journals.openedition.org/ifha/243 ; DOI : 10.4000/ifha.243

Ce document a été généré automatiquement le 1 mai 2019.

(C)IFHA 


\title{
L'historiographie germanophone sur les lois barbares : centres de gravité, évolutions, desiderata
}

\author{
Thomas Lienhard
}

1 Dès la fin de l'Antiquité, des princes d'origine barbare récemment installés en Europe commencèrent à promulguer des textes législatifs ; s'inspirant en cela du modèle impérial romain, ils furent à leur tour imités par leurs successeurs, de sorte que, entre le Ve et la fin du IXe siècle, se constitua un vaste corpus législatif que l'historiographie rassembla sous l'appellation de "lois barbares». Les auteurs de ces lois espéraient assurément influencer l'ordre social de leur temps; en revanche, ils ne se rendaient probablement pas compte que leur œuvre allait également agiter le milieu des historiens quelque 1500 ans plus tard. Il est vrai que, pour celui qui s'intéresse aux sociétés tardo-antiques, ces textes législatifs présentent une importance cruciale : non seulement ils contiennent la clé de la rationalité normative qui gouvernait leur époque, mais ils constituent également une source incontournable pour répondre à d'autres questions familières aux hautsmédiévistes, parmi lesquelles la continuité entre l'Antiquité et le Moyen Âge, la nature des premiers peuples médiévaux ou encore le développement du pouvoir princier et des autorités publiques. De ce fait, l'intérêt pour ce sujet reste vif aujourd'hui, en particulier dans l'historiographie germanophone. Celle-ci, en effet, présente quelques spécificités notables en ce domaine, initialement en raison du caractère germanique qui fut d'abord attribué à ces lois et qui, de ce fait, intéressa l'école romantique ; mais encore aujourd'hui, les études allemandes en ce domaine se distinguent par leur intensité, par leurs institutions et par la nature de leurs résultats. C'est là une première raison pour présenter ce thème dans la Revue de l'IFHA, toujours soucieuse de faire connaître au public francophone les caractéristiques de la recherche historique d'outre-Rhin.

2 La seconde raison réside dans le regain d'actualité historiographique que connaît depuis peu, dans ces pays, le thème des lois barbares: le vieux chêne de l'histoire du droit allemande a tendance à plier et, simultanément, à produire de nouvelles pousses. Les causes d'un tel rajeunissement, toujours partiellement énigmatiques, sont en tout cas 
diverses : elles sont liées à la publication récente de nombreux outils de travail, à des colloques de synthèse sur des thématiques qui étaient indécises depuis bien longtemps, ou encore à l'osmose croissante entre les historiens et les historiens du droit (deux catégories traditionnellement distinctes en Allemagne) et, par là, à la timide pénétration de thématiques allogènes et interdisciplinaires dans la vénérable forteresse juridicohistorique.

Ce sont ces tendances récentes que l'on analysera ici en plusieurs temps ${ }^{1}$. On présentera d'abord le paysage institutionnel allemand en matière de recherches sur les lois barbares, car ce point peut non seulement intéresser le chercheur soucieux de coopération avec ses collègues germanophones, mais constituer également un facteur d'explication en tant que tel; puis on présentera les résultats de l'historiographie germanophone sur des thèmes renouvelés récemment, en particulier l'origine des lois barbares, le public auquel celles-ci étaient destinées, ou encore l'utilité pratique qu'elles pouvaient présenter ou non ; enfin, on esquissera un tableau des questions nouvelles, voire encore balbutiantes, qui diversifient aujourd'hui les recherches germaniques sur le droit altimédiéval.

4 L'historiographie allemande à propos des lois barbares se distingue d'abord par l'abondance de ses institutions, fidèle reflet de l'intérêt multiséculaire que l'Allemagne a porté à l'histoire du droit. À ce sujet, on ne peut faire l'économie d'une référence à Friedrich Carl von Savigny (1779-1861), dont le modèle juridique fut élaboré en grande partie en opposition à la Révolution française et au Premier empire. Face à la politique de table rase juridique promue à Paris, Savigny théorisa le modèle d'un droit fortement ancré dans le passé ; et contrairement aux aspirations universalistes du Code civil, le savant allemand considéra que le droit était l'une des expressions les plus fidèles de l'esprit spécifique d'un peuple. Le romantisme allemand s'engouffra dans cette double brèche et donna lieu à une abondante production historiographique, alors qu'en France, l'école historique du droit ne trouva pas son Victor Hugo, marquée de surcroît par le soupçon d'accointance intellectuelle avec l'ennemi germanique ${ }^{2}$.

5 Conséquence fidèle de ces anciennes prémices, la densité des centres de recherche qui se consacrent à l'histoire du droit reste impressionnante dans l'Allemagne actuelle. Rares sont les grandes universités qui n'hébergent pas un Institut für Rechtsgeschichte; Munich abrite même deux de ces établissements, qui comptent d'ailleurs parmi les plus importants en histoire du droit altimédiéval, à savoir le Leopold-Wenger-Instituts für Rechtsgeschichte et le Stephan Kuttner Institute of Medieval Canon Law. De son côté, la SavignyStiftung prolonge jusqu'à nos jours, mutatis mutandis, les vues du savant romantique qui fonda l'école historique du droit, éditant en particulier une revue considérée comme une référence internationale ${ }^{3}$. Il est vrai que depuis 1964, elle trouve une concurrente importante en la puissante société Max-Planck, puisque celle-ci créa alors à Francfort le Max-Planck Institut für Europäische Rechtsgeschichte: ce dernier est un des principaux acteurs de cette scène intellectuelle, hébergeant une des plus belles bibliothèques d'Europe en ce domaine, produisant lui aussi une revue désormais incontournable ${ }^{4}$, alimentant un collège de recherche international en histoire du droit comparée et constituant ainsi le dernier maillon de cette chaîne institutionnelle. Tout en cultivant leur autonomie et leur ligne éditoriale spécifique, ces établissements sont régulièrement reliés par des manifestations fédératrices : outre l'imposant Deutscher Rechtshistorikertag, qui réunit tous les deux ans les spécialistes mondiaux du sujet, on peut noter également la forte participation des jeunes chercheurs allemands au European Forum of Young Legal Historians. On perçoit ainsi l'intensité du réseau qui maille les recherches allemandes en 
histoire du droit, dont une partie considérable est consacrée (comme toujours dans l'historiographie allemande) au haut Moyen Âge qui nous occupe; on ne sera donc pas surpris d'apprendre que le nombre des spécialistes germanophones des lois barbares s'élève aujourd'hui à une quarantaine, soit quatre à cinq fois plus qu'en France.

6 Si l'on se tourne à présent vers la formation et la carrière de ces chercheurs, une autre originalité allemande s'impose d'évidence: non seulement les historiens du droit constituent une catégorie nettement distincte par rapport à celle des autres historiens, comme on le suggérait plus haut, mais pour une grande part d'entre eux, ils ont été juristes avant de se tourner vers l'histoire. Il est vrai que cet état de fait est facilité par l'existence de passerelles thématiques, et à cette occasion, il faut rendre hommage à l'ouverture d'esprit des facultés de droit germaniques, qui distribuent sans hésiter des sujets de thèse ou d'habilitation portant sur la législation de Justinien, sur le droit canon médiéval ou sur l'origine sociale du patriciat allemand. Mais il n'est pas exclu non plus que cette particularité sociologique des historiens du droit ait contribué à polariser leurs recherches scientifiques: sans vouloir abuser de l'argument institutionnel, on peut estimer qu'un juriste est plus facilement tenté de considérer les textes légaux d'une époque révolue comme les précurseurs des outils de travail qu'il a lui-même maniés à l'époque contemporaine : de nombreux angles de recherche appliqués aux lois barbares, tels que «l'origine de l'ordre public » ou " la genèse du droit privé moderne », peuvent s'expliquer par ce biais. À cette première sectorisation institutionnelle s'en ajoute une autre, à savoir l'importance de l'histoire régionale: ce furent majoritairement des laboratoires de Bavière qui analysèrent la loi des Bavarois, et ainsi de suite. Or cette Landesgeschichte postule, par définition, une certaine linéarité historique de l'espace qu'elle envisage, renforçant ainsi la tendance qui consistait à ne voir dans le droit altimédiéval qu'un prototype de la situation moderne.

7 Il faut toutefois noter une inversion récente de cette sociologie des chercheurs, puisque depuis une vingtaine d'années, les spécialistes de la législation (alti)médiévale ont suivi prioritairement une formation d'historiens. Il est possible que, de ce fait, ces nouveaux historiens du droit aient une connaissance pratique moins développée des textes législatifs (et leurs aînés ne se privent pas de le suggérer); en revanche, ils manifestent une plus grande sensibilité pour les thématiques sociales et culturelles qui se sont développées dans les autres branches de l'historiographie européenne durant les dernières décennies. Les données institutionnelles constituent ainsi un facteur parmi d'autres pour expliquer la mue de la recherche allemande, qui aborde aujourd'hui les lois barbares avec une intensité conservée, mais des thématiques renouvelées.

Dans certains cas, le renouveau historiographique a simplement consisté à aborder de manière plus fine et plus critique des problématiques déjà anciennes; il est vrai que ce supplément de soin suffisait parfois à produire des résultats très novateurs. Tel est le cas notamment des études qui portaient sur la question, classique mais incontournable, des origines des lois barbares: quand, par qui et avec quels outils ces textes furent-ils promulgués?

9 C'est peut-être en termes de datation que les soubresauts récents de l'historiographie ont le plus malmené ce qu'on croyait savoir auparavant. Rares sont, en effet, les codes de lois barbares pour lesquels l'historien dispose d'un indice simple de datation : tel n'est le cas, à l'heure actuelle, que pour les lois des Anglo-Saxons et celles des Lombards, dans lesquelles le copiste a pris soin d'inscrire une date précise qui est confirmée, dans le second cas, par la tradition manuscrite ${ }^{5}$. Dans les autres cas en revanche, qui sont 
majoritaires, la datation traditionnelle repose sur une mention évasive glanée dans le prologue ou l'épilogue d'une compilation tardive. On admet ainsi généralement que le Pactus Alamannorum remonte au début du VIIe siècle, parce que, dans un texte postérieur qui se fondait sur ce corpus, on mentionnait l'activité législative de Clotaire II (613-629) ${ }^{6}$. Mais cette conception fut considérablement malmenée dans les dernières années: l'auteur médiéval qui avait nommé ce prince était-il fiable? Et son projet consistait-il bien à établir l'historique du Pactus en particulier, ou bien cherchait-t-il plus généralement, en guise d'introduction, à énumérer quelques étapes importantes dans l'histoire du droit, tout comme Isidore de Séville, avant lui, avait dressé un panorama des grands législateurs depuis Moïse ${ }^{7}$ ?

10 Un autre argument fragile de datation repose sur une conception évolutionniste de l'histoire: des spécialistes ont ainsi considéré que la loi salique ne pouvait avoir été rédigée dans les dernières années du règne de Clovis, car son horizon très modeste, essentiellement cantonné à une exploitation agricole, ne correspondrait pas au degré d'extension et d'organisation du royaume franc après $507^{8}$. Un tel schéma, on le voit, est périlleux : non seulement un texte littéraire, y compris législatif, peut délibérément se cantonner à un secteur social bien précis même si la société de son temps est plus complexe, mais par ailleurs, rien, dans l'histoire antique ou médiévale, ne permet d'exclure l'expansion territoriale d'un groupe qui aurait été composé essentiellement de paysans modestes. C'est avec ces arguments évanescents que l'historiographie germanophone prend progressivement ses distances depuis quelques années.

11 Pour compliquer le tout, les lois barbares sont notoirement des compilations, dont les différents ingrédients n'ont pas toujours été homogénéisés ni adaptés à la société de celui qui les rassemblait. De ce fait, ces éléments épars présentent parfois entre eux des contradictions chronologiques, sensibles notamment dans les incohérences du système monétaire présenté dans tel ou tel corpus ${ }^{9}$. Mais surtout, cette composition hétéroclite rend le texte très difficile à dater : dans quelle mesure le compilateur a-t-il fait œuvre originale, ou a-t-il respecté au contraire la version originelle des éléments qu'il rassemblait? C'est souvent sur ce facteur de confiance que reposent aujourd'hui les écarts entre spécialistes à propos de la datation d'une loi barbare ${ }^{10}$. Plus généralement, les avis restent aujourd'hui divergents entre les historiens sceptiques et ceux qui sont plus enclins à se fier, faute de mieux, à des indices parfois fragiles; mais la tendance générale de l'historiographie récente consiste à abandonner des datations trop précises, mettant ainsi l'historien dans la situation très juste mais très inconfortable consistant à devoir étudier un texte sans pouvoir dater celui-ci plus précisément que par une fourchette de deux siècles.

12 Par-delà la question de la date, c'est également la nature des auteurs de ces lois qui continue de diviser la communauté des historiens germanophones. Et notamment, sur ce terrain comme pour d'autres données tardo-antiques, les spécialistes croisent le fer pour déterminer quelle part accorder, dans la genèse des lois barbares, à l'élément germanique, où au contraire à l'héritage romain éventuellement transformé par des évolutions locales. La question n'est pas nouvelle, puisqu'elle remonte au moins à Savigny et à ses disciples; ces derniers, encore plus intensément que leur maître, avaient forgé le modèle d'une forte influence germanique ${ }^{11}$. Mais récemment, en relation avec d'autres études sur la composition ethnique des populations médiévales ${ }^{12}$, ce thème a connu un regain d'actualité. Signalons toutefois qu'en l'occurrence, les opinions restent très diverses, sans que l'on puisse actuellement désigner un vainqueur dans ce match 
historiographique ; tout au plus peut-on opposer un schéma classique et des tendances plus récentes, même si celles-ci, à l'heure actuelle, sont encore loin de faire l'unanimité.

Comme vitrine de ces débats, on peut rappeler notamment les résultats du colloque " Leges - Gentes - Regna ", qui fut tenu sur ce thème en Bavière en 2004 et dont les actes furent publiés deux ans plus tard ${ }^{13}$. Dans sa conférence introductive, Gerhard Dilcher présenta un schéma qui était relativement classique au sein de l'historiographie germanophone. Certes, il prit ses distances avec le projet de ses prédécesseurs qui avaient cru possible de retrouver, par un travail de comparaison et de reconstitution, un droit originel commun dont seraient issues toutes les législations germaniques; il estima pourtant que les Germains, s'installant dans l'empire romain à la fin de l'Antiquité, apportèrent une forte contribution originale au droit occidental. En particulier, le phénomène de la faide ${ }^{14}$, ainsi que le système de composition développé en opposition à cette dernière, auraient été typiquement germaniques. Cette législation aurait d'ailleurs été d'autant plus représentative pour ces sociétés qu'elle aurait été non pas édictée par quelques princes plus ou moins éclairés, mais bien adoptée en commun par un groupe d'experts juridiques suffisamment nombreux pour représenter fidèlement les aspirations des Germains récemment parvenus dans l'empire ${ }^{15}$. Enfin, pour expliquer cette forte influence malgré l'absence de toute loi écrite antérieure chez les Germains, G. Dilcher fit sienne l'hypothèse classique selon laquelle, avant le milieu du Ve siècle, ces peuples auraient disposé d'un droit qui, quoique nettement structuré, n'aurait existé que sous forme orale. Cet apport germanique permettrait notamment d'expliquer l'écart entre les lois barbares et le droit romain classique ou le Code Justinien. De cette communication, le droit germanique sortait renforcé ${ }^{16}$.

D'autres intervenants en revanche, corroborés entre-temps par diverses publications parues après ce colloque, écornèrent ce schéma. En particulier, plusieurs études mirent en lumière la relation étroite qui, contrairement aux postulats de l'école germaniste, avait subsisté entre les lois barbares et le droit romain. Il est vrai que celui-ci constituait lui-même une galaxie très vaste, et que ses composantes les plus connues, notamment les étapes théodosiennes et justiniennes, influencèrent assez peu les textes législatifs de l'Europe post-romaine. En revanche, le droit romain vulgaire, déjà identifié par Ludwig Mitteis et Ernst Levy au tournant du XXe siècle, fut fréquemment utilisé au haut Moyen Âge, et plusieurs publications récentes soulignent l'importance de ce chaînon manquant entre Rome et les législateurs barbares ${ }^{17}$. La tradition manuscrite du droit romain luimême, en particulier du code Justinien, fait d'ailleurs actuellement l'objet de révisions, qui réévaluent son importance dans les sociétés occidentales du haut Moyen Âge ${ }^{18}$. Certes, ce corpus n'était pas toujours exploité dans les nouvelles législations, qui le prenaient même parfois radicalement à contre-pied; mais, de manière frappante, il était négligé tout particulièrement dans les régions où sa bonne conservation manuscrite est nettement attestée, produisant souvent l'impression que les législateurs le passaient sous silence non pas parce qu'ils l'ignoraient, mais précisément parce que, le considérant comme connu et disponible, ils jugeaient plus utile d'explorer des voies juridiques complémentaires ou alternatives ${ }^{19}$. Au terme de cette revalorisation romaine, le caractère germanique du droit altimédiéval ne constitue plus une nécessité logique pour expliquer les lois barbares.

15 Par ailleurs, plusieurs éléments qui étaient reconnus, encore naguère, comme les plus caractéristiques pour une origine germanique des lois virent récemment ce statut exotique remis en question. Tel est le cas, en particulier, pour la faide ${ }^{20}$. Certes inconnue 
dans le monde romain, cette forme de compensation directe entre la victime et le coupable ne constitue pas non plus l'apanage des sociétés germaniques à la fin de l'Antiquité : il est donc légitime d'envisager, comme explication pour cette nouveauté, soit une importation à partir d'autres cultures que celle des Germains, soit également une évolution interne, sans apport extérieur, qui aurait affecté l'Europe occidentale au début du Moyen Âge comme elle le fit également dans d'autres régions du monde à des époques plus tardives. De même, des réflexions similaires ont fait évoluer le statut de preuve que possédaient les gloses dites "malbergiques", ces annotations en langue germanique insérées dans la loi salique. À leur sujet, l'interprétation habituelle est la suivante ${ }^{21}$ : elles sont généralement considérées comme les témoins de concepts juridiques déjà employés par les populations germanophones avant la rédaction des lois barbares, et on admet fréquemment qu'elles auraient été intégrées dans cette nouvelle législation, pourtant écrites en latin, pour ne rien perdre en précision par rapport à ce droit antérieur. Elles constitueraient ainsi une preuve de l'antériorité d'un droit germanique, et, du même coup, une aubaine pour les linguistes qui disposeraient ainsi d'un des plus anciens corpus lexicaux germaniques ${ }^{22}$. Rien n'empêche cependant de renverser le schéma, d'envisager l'antériorité de la version latine, et de considérer alors que ces gloses germaniques constituaient des éléments pédagogiques destinés à éclairer, à propos de la loi écrite, un auditoire ou des lecteurs familiers $d u$ francique; cette hypothèse implique certes l'existence de populations qui étaient encore germanophones, mais ne nécessite plus de penser que ces dernières eurent un privilège d'antériorité par rapport aux rédacteurs de la loi salique ${ }^{23}$. Au terme de ces débats, on ne peut toujours pas exclure que les nouveaux arrivants en Europe aient apporté leur pierre à l'évolution juridique du haut Moyen Âge, mais celui qui veut prouver une telle influence sent désormais le sol se dérober sous les pieds : ce sont des atteintes discrètes mais efficaces qui ont été portées récemment au Léviathan savignien du droit germanique.

Si les modèles concernant les auteurs des lois se voient éroder peu à peu, c'est un renversement beaucoup plus radical qui a affecté récemment les conceptions des historiens allemands à propos du public auquel étaient destinées les lois barbares. Plus précisément, c'est le schéma de la personnalité des lois qui n'est désormais plus défendu que par une minorité de spécialistes. De quoi s'agit-il ? Selon le concept traditionnel à ce sujet, les habitants d'un même territoire politique auraient été soumis, au haut Moyen Âge, à des droits concurrents, en fonction de leurs origines ou des classifications ethniques subies après leur naissance : les Francs auraient dépendu de la loi salique, les Burgondes (même s'ils résidaient sur le même territoire que les précédents) auraient été gouvernés par la loi Gombette, et ainsi de suite. Un cas particulier, mais qui est le plus souvent invoqué par les tenants de cette personnalité des lois, est à chercher dans les «lois romano-barbares» (leges Romanae): étant donné la coexistence de fait, durant l'Antiquité tardive, entre les anciennes populations de l'empire romain et les nouveaux arrivants d'origine germanique, les historiens ont admis naguère que les royaumes postromains auraient parfois produit un code de loi distinct pour chacun de ces deux groupes. En Bourgogne, par exemple, la loi Gombette aurait prévalu pour le groupe des Burgondes récemment installés sur place, tandis qu'une " loi romano-burgonde » aurait été destinée aux Gallo-Romains du même territoire. La situation législative du haut Moyen Âge aurait donc été celle d'une mosaïque complexe de droits, face à laquelle une logique d'unification territoriale ne se serait imposée qu'après plusieurs siècles. Tel est le tableau qui semble encore émerger d'un pamphlet d'Agobard de Lyon (778-840), dans lequel l'archevêque déplorait la persistance, pour certaines de ces ouailles, de la loi Gombette 
alors que régnait déjà majoritairement la loi salique, amenant ainsi parfois les membres d'une même maison à relever de droits différents ${ }^{24}$. Ce schéma classique, que l'on trouve encore représenté dans quelques publications ${ }^{25}$, était quasiment consensuel il y a encore vingt ans.

Dans l'intervalle, toutefois, cette belle construction intellectuelle a été démontée pierre par pierre. Les premières remises en cause ont semblé presque anodines, ne portant d'abord que sur le titre des codes de loi, qui semblait suggérer, à première vue, une partition juridique sur une base ethnique : si un corpus législatif wisigoth portait le nom de lex Romana, n'était-ce pas parce qu'il était destiné à la part romaine de la population? Selon les contributions les plus récentes, la réponse est clairement négative: dans la grande majorité des sources (y compris wisigothiques), ces termes ne désignent rien d'autre que le droit romain classique, sans distribution ethnique particulière ${ }^{26}$. Par ailleurs, l'historiographie a également fait remarquer que les titres des lois barbares, qui contenaient encore divers autres ethnonymes, relevaient souvent d'un archaïsme. Pour prendre un exemple particulièrement net, à l'époque où fut conçue la loi "salique », le nom des Saliens avait déjà disparu du discours public depuis plusieurs décennies, et témoignait donc d'un raffinement par anachronisme plutôt que d'une volonté de distinguer un quelconque groupe ethnique qui, dans tous les cas, ne portait plus ce nom depuis longtemps ${ }^{27}$. Le titre des codes législatifs voyait ainsi s'évaporer sa valeur argumentative pour les publics concernés par la loi ; il est vrai que cet argument n'ayant jamais été présenté comme décisif auparavant, ce premier renversement historiographique ne fut pas encore déterminant.

Le modèle de la personnalité des lois eut davantage à souffrir lorsque, dans le corps même des codes barbares, des études récentes éliminèrent l'un après l'autre les exemples qui auraient pu nourrir (et qui avaient nourri) l'idée d'une partition légale sur une base ethnique. En particulier, il fut observé que dans la législation lombarde, la distinction entre Germains et Romains étaient particulièrement abstraite et, par conséquent, peu propice à une application judiciaire ${ }^{28}$. De fait, les rares cas pratiques que l'on avait souvent cités comme illustrations d'une concurrence législative à fondement ethnique, par exemple un Goth revendiquant un droit spécifique au sein du royaume lombard, furent démasqués comme des interpolations ${ }^{29}$. Les doutes se renforcèrent encore lorsque l'on rappela que par ailleurs, les lois lombardes s'attribuaient une validité territoriale, concernant les descendants des anciennes familles locales au même titre que les immigrants de fraîche date ${ }^{30}$. À partir de ce modèle lombard, la remise en question fut étendue à d'autres corpus, en particulier à la loi des Alamans. Pour expliquer la présence manuscrite de celle-ci en Italie du Nord à une date précoce, l'historiographie estimait naguère qu'il fallait envisager des minorités d'Alamans dans cet espace dès le milieu du VIIIe siècle, qui auraient recouru à cette législation contre les lois lombardes ${ }^{31}$. Les contradicteurs récents ont pourtant rappelé que la tradition manuscrite ne permettait pas de savoir si ces textes avaient été introduits en Italie avant ou après la conquête franque, et que l'on pouvait donc très bien attribuer leur importation à l'administration carolingienne qui, activée à construire une réforme législative à la fin du VIIIe siècle, a vraisemblablement importé en Italie, à titre documentaire, toutes sortes de corpus légaux y compris celui-ci. Ce point de vue est d'autant plus probable que les manuscrits conservés sont généralement des collections de $\operatorname{lois}^{32}$ : la cohabitation de la loi des Alamans et des lois lombardes en un même espace aurait alors répondu à une logique non 
pas ethnique, mais bibliothécaire. Ainsi, l'examen du corps même des lois multipliait les nouveaux arguments contre une distinction ethnique altimédiévale.

Dans ces conditions, comment l'historiographie actuelle explique-t-elle la coexistence incontestable de droits divers en un même royaume, comme la dépeignait Agobard? Outre le cas particulier des collections manuscrites présentées à l'instant, il semble que la logique de ce chevelu juridique consistait non pas à tenir compte de l'identité ethnique des populations concernées, mais à distinguer entre diverses forces de loi ou à compléter des lacunes du droit. Ainsi, dans le cas du royaume wisigothique, il a été noté que l'existence de deux lois parallèles ne correspondait pas à des publics distincts, car l'une et l'autre de ces législations prennent en compte la population romane ${ }^{33}$; en revanche, les sources font une distinction nette entre les lois qui sont explicitement d'origine royale et la loi romano-wisigothique (le "Bréviaire d'Alaric ») qui revendique ouvertement des sources d'inspiration diverses. Peut-être s'agissait-il ainsi de distinguer des genres littéraires distincts; peut-être est-il permis d'aller plus loin et de considérer que, dans une perspective plus pratique, il s'agissait de donner une force de loi différenciée à ces deux types de textes.

Dans d'autres cas, la rédaction d'une seconde loi visait clairement à compléter des absences dans le corpus législatif antérieur; un tel complément était d'autant plus important que, vu la brièveté des codes de lois barbares, la casuistique complexe du Corpus Iuris Civilis avait été partiellement oubliée, nécessitant un nouvel éclairage à propos de certains délits. Ainsi, on a pu constater que sur bien des thèmes, la loi romanoburgonde, loin d'entrer en conflit avec la loi Gombette en vigueur dans le même espace, envisageait les cas que cette dernière n'avait pas pris en compte, en particulier les éléments de droit commercial ${ }^{34}$. Il est vrai que, dans le cas particulier de cet espace burgonde, ce parallélisme semble bien avoir impliqué également une distinction entre plusieurs publics: l'historiographie la plus récente continue d'admettre que la loi romano-burgonde concernait essentiellement les Burgondes, les autres barbares et les juifs, à l'exclusion des Gallo-Romains chrétiens de ce royaume ${ }^{35}$. Il y aurait donc bien eu, en l'occurrence, des lois différentes selon l'identité des justiciables, mais il faut relever également que cette distinction ne se faisait pas sur une base ethnique simple, puisque par exemple, une famille juive anciennement soumise au droit romain se voyait classer dans la même catégorie qu'un Burgonde récemment installé dans l'empire. On ne peut d'ailleurs s'empêcher d'être frappé par le fait que la liste des groupes concernés par cette loi romano-burgonde est précisément celle des populations qui n'étaient pas soumises au droit ecclésiastique: dans ces conditions, il semble vraisemblable que la logique de ce texte consistait à étendre le corpus normatif à des populations particulières, et non pas à tenir compte d'une spécificité ethnique antérieure. Au final, l'existence de lois parallèles reste admise, mais avait probablement pour fonction essentielle de tenir compte de registres divers (en fonction de l'auteur, en fonction du type de droit), de manière à compléter un système juridique. L'historiographie récente a d'ailleurs admis que cette interpénétration légale avait contribué à la distinction moderne entre le droit privé et le droit public ${ }^{36}$; en revanche, une concurrence directe entre des droits opposés, par exemple sur une base ethnique, semble aujourd'hui fort peu probable.

21 Si la notion de droit ethnique se voit ainsi contredite par les sources, signalons toutefois, à la décharge des historiens qui avaient développé ce schéma au XIXe et au XXe siècle, que ces derniers ne furent pas les premiers à mésinterpréter la coexistence des lois altimédiévales. Déjà à l'époque carolingienne, on peut déceler des indices qui montrent 
que les copistes ne savaient plus quel pouvait être l'usage pratique de cette diversité légale mérovingienne. Ce point est sensible à travers la tradition manuscrite. La loi romano-wisigothique, en particulier, fut d'abord transmise dans des codex qui contenaient divers textes romains brefs ou résumés, ce qui correspondait bien à sa fonction originelle; mais à partir du VIIIe siècle, elle fut recopiée conjointement avec des lois barbares, se voyant ainsi classée, dans l'esprit des scribes carolingiens, comme une création originale destinée à un peuple spécifique, et non plus comme un complément. Ce fut donc dès le haut Moyen Âge, mais a posteriori, que les lois barbares se virent attribuer une portée ethnique que rien ne peut prouver pour les Ve et VIe siècles.

Il est vrai que sur un point, les études récentes donnent raison à ce malentendu. Il semble en effet possible, à nouveau, d'envisager un lien entre la loi et l'ethnicité, mais en renversant le schéma historiographique initial: les textes légaux altimédiévaux ne reflétaient pas une identité ethnique, mais ils ont pu contribuer à créer celle-ci. Tel fut le cas en particulier pour les Ripuaires. À propos de ces derniers, il a été montré que leurs noms n'apparaissaient pas dans les textes historiographiques ou juridiques avant le VIIIe siècle. En revanche, dès le VIe ou le VIIe siècle, l'élaboration de la loi des Ripuaires produisit un effet ambigu: d'une part, ce texte, fortement influencé par la loi salique, contribuait à intégrer les Ripuaires dans l'ensemble juridique franc, mais d'autre part, fixant par écrit quelques spécificités, il donnait simultanément à ce groupe une définition autonome. On perçoit là l'ampleur du renversement connu par l'historiographie germanophone à propos du droit altimédiéval : renonçant à l'idée d'une concurrence ethnique originelle qui aurait été progressivement surmontée par une rationalité inspirée de Rome, les spécialistes actuels, en osmose avec le modèle international récent du " making of... », envisagent aujourd'hui une création progressive d'identité qui, de manière tardive et administrative, a fini par injecter un sens ethnique dans la multiplicité des droits qui était en vigueur sous l'empire romain. Savigny est aujourd'hui bien loin.

À ces deux premières remises en question s'en est ajoutée une troisième, dont le sujet était encore plus radical. Il s'agissait en effet de déterminer non seulement à quel degré les lois barbares furent appliquées, mais également dans quelle mesure elles étaient réellement destinées à l'être. La question peut surprendre celui qui n'est pas familier du haut Moyen Âge. Quoi! Des milliers de pages à caractère juridique auraient été rédigées sans souci d'application judiciaire? C'est pourtant là l'hypothèse qui a été formulée par Hermann Nehlsen en 1977, et qui, depuis, nourrit une discussion continue parmi les spécialistes allemands ${ }^{37}$. L'argumentation de Nehlsen était la suivante : s'appuyant sur le caractère à la fois lacunaire et contradictoire des lois barbares sous leur forme conservée, l'historien considéra qu'il s'agissait là d'une adaptation maladroite du droit romain. Le degré d'imperfection, selon lui, était tel qu'il fallait chercher dans ces textes un autre objectif que le souci d'application directe. Dans cette hypothèse, l'activité législative aurait d'abord constitué une posture politique, destinée non pas à faciliter une cohérence judiciaire mais à valoriser le législateur: dans le contexte du VIe siècle qui vit les monarchies barbares rechercher l'autonomie par rapport à Byzance, les princes auraient singé la législation impériale pour appuyer leurs prétentions au pouvoir. Enfin, Nehlsen expliquait le caractère extrême ou déroutant de certaines peines par le fait que ces lois, à supposer qu'elles aient visé une application quelconque, constituaient non pas le droit de première instance, mais l'ultime recours judiciaire: la sévérité des peines aurait été destinée à contraindre les parties judiciaires à s'accorder entre elles avant de faire appel à une telle loi ${ }^{38}$. 
Tel était le point de vue de Nehlsen il y a une trentaine d'années; dans l'intervalle, d'autres voix se sont fait entendre, s'efforçant de restaurer la crédibilité pratique des lois barbares. Où en est l'historiographie aujourd'hui ? Précisons immédiatement que les réponses disponibles dans l'historiographie actuelle sont fort différenciées, d'abord parce que, au sein de ces lois altimédiévales, la gamme des situations possibles est très large. En effet, dans le cas des lois des Lombards, une rédaction ordonnée, des mesures rigoureuses de validation visant à exclure des versions incorrectes de la loi, et une excellente tradition manuscrite conduisent la grande majorité des spécialistes à admettre que cette loi fut conçue pour être appliquée; à l'inverse, à propos de la Lex Romana Curiensis, la plupart des historiens du droit considèrent qu'il ne peut s'agir que d'un texte inachevé, voire de l'œuvre d'un juriste incompétent ${ }^{39}$. À défaut d'une étude au cas par cas, il faudrait sans doute respecter une différenciation chronologique qui distinguerait les premiers codes de loi du Ve et du VIe siècle, la vague de législation volontariste sous Charlemagne, et les textes du haut Moyen Âge post-carolingien : pour ces derniers, à tout le moins, l'historiographie est unanime pour considérer que, pâles répliques d'un système disparu, ils ne pouvaient plus prétendre à aucune effectivité. Mais pour les premiers siècles du Moyen Âge, l'historiographie n'a fourni ni réponse tranchée, ni synthèse à ce sujet depuis $\mathrm{H}$. Nehlsen : on ne pourra donc proposer qu'une présentation ordonnée des informations fragmentaires glanées ça et là à ce sujet. Enfin, on se permettra ici de rester relativement impressionniste sur cette question, dans la mesure où une étude plus complète est en préparation ${ }^{40}$.

Pour les chercheurs qui souhaitaient sonder l'effectivité des lois barbares, un réflexe de bonne méthode consista à éviter une analyse strictement interne de ces textes, et à se tourner d'abord vers les informations fournies par d'autres types de sources. Or en ce domaine, les historiens furent vite déçus : que ce soit dans les sources narratives ou dans les actes de la pratique, les références à la lex scripta, qui auraient permis de déterminer si celle-ci était prise en compte ou non au quotidien, étaient rares, et lorsqu'elles existaient, leur leçon était contradictoire. Dans certains cas, en effet, ces sources complémentaires confirmaient l'application de la loi, y compris pour les rituels qui déroutent le plus l'historien moderne ${ }^{41}$; dans d'autres cas au contraire, et même dans les diplômes qui constituaient pourtant une source à caractère public, elles attestaient que la pratique allait parfois radicalement à l'encontre de la loi royale ${ }^{42}$. Cette première approche fut donc peu déterminante pour la question de l'effectivité.

En revanche, les textes de loi eux-mêmes fournissaient des indices abondants pour démontrer un souci d'application pratique de la part du législateur et, dans certains cas, une application pratique réelle; c'est donc là que l'historiographie a pu récolter quelques arguments pour démontrer que les copistes altimédiévaux considéraient bien ces textes comme des outils de travail, et non seulement comme un exercice littéraire ou une source de prestige pour le prince. Tel est d'abord le cas de la codicologie : à côté des manuscrits d'apparat, en effet, les lois barbares sont fréquemment représentées dans des formats typiques pour un usage courant, qui pouvaient aller jusqu'à un simple cahier ou même une simple feuille volante contenant les désinformations prioritaires pour le propriétaire de l'exemplaire ${ }^{43}$. Par ailleurs, l'organisation du texte comporte souvent des outils destinés à faciliter un travail de lecture rapide ou d'application aisée : ainsi, plusieurs manuscrits contiennent des données synthétiques permettant de résumer un corpus très vaste, ou encore des véritables concordances ${ }^{44}$. Dans d'autres cas, le copiste a inséré des règles de calcul facilitant les conversions pour le Wergeld, typique pour ces lois, et 
d'autres types de composition ${ }^{45}$. Tous ces éléments furent relevés par l'historiographie comme les signes d'un discours à finalité pratique, et non pas comme un simple exercice de style. Signalons toutefois que ce premier résultat ne résout qu'en partie la question de l'effectivité : certes, il s'agissait incontestablement là d'outils de travail, mais étaient-ils destinés à des juges, ou à des bibliothécaires chargés de rassembler les lois à des fins documentaires? Dans la mesure où, comme on le verra, les manuscrits qui nous ont transmis les lois barbares reflétaient souvent un souci d'accumulation d'information dans une logique d'archives, ce soupçon ne peut être écarté complètement, et cette série d'indices ne permet pas encore d'inférer avec certitude un objectif d'application pratique des lois au tribunal.

Dans certains cas, il semble même certain que le manuscrit était destiné à une collection de type encyclopédique, à l'exclusion d'un objectif pratique. C'est ainsi, notamment, que l'historiographie a mis en exergue des manuscrits du Xe siècle qui rassemblaient la loi des Saxons et la loi des Thuringiens, sans qu'aucun séparateur visuel ne permît une distinction entre ces deux textes, alors que ceux-ci étaient en partie incompatibles ${ }^{46}$. Il est vrai que ce même manuscrit marquait soigneusement la différence, en revanche, entre les données juridiques et d'autres renseignements, par exemple d'ordre théologique: manifestement, une telle classification était encore considérée comme utile, peut-être dans l'espoir de pouvoir ré-exploiter ces textes par la suite pour une nouvelle entreprise législatrice. Mais dans l'immédiat, les textes de loi étaient ici rassemblés de manière éparse, ne pouvant constituer un outil de travail pour le juge, puisque celui-ci aurait été gêné par l'existence de clauses divergentes sur un même sujet. Il s'agit certes là d'un cas tardif, à une époque à laquelle le contexte ne se prêtait plus à l'application des lois carolingiennes ; mais il n'est pas exclu que les lois barbares de l'époque classique, c'est-àdire antérieures au milieu du IXe siècle, aient également constitué de telles collections à fonction documentaire plutôt que judiciaire. On a observé, en particulier, de fortes contradictions dans la loi des Bavarois qui, pour le cas d'un duel judiciaire, prescrivait une sélection des combattants par tirage au sort alors que dans d'autres clauses, elle interdisait cette pratique ${ }^{47}$; de même, la loi des Frisons autorisait le meurtre des enfants en une occurrence alors qu'elle l'interdisait ailleurs ${ }^{48}$. De telles incohérences peuvent s'expliquer par le recours à des sources d'informations multiples, compilées sans souci d'homogénéité ni, par conséquent, d'effectivité.

Inversement, ce souci documentaire permet d'exonérer les lois barbares d'un grief qui leur fut souvent adressé par les historiens soucieux d'efficacité juridique. Rappelons en effet que pour Savigny, suivi en cela par de nombreux spécialistes, plusieurs lois altimédiévales ne pouvaient être appliquées en l'état, parce que trop lacunaires; ce courant de l'historiographie considérait ces textes comme un méli-mélo d'occurrences trouvées au gré d'autres sources, qui portait la double tare de n'être ni complet, ni systématique, rendant ainsi une application impossible ${ }^{49}$. Plus récemment, l'historiographie est pourtant revenue de cette opinion, rappelant en effet que, les princes altimédiévaux revendiquant très rarement le monopole de la production législative, il serait anachronique de chercher un système normatif complet dans une seule loi : il est bien préférable de penser en termes de complémentarité entre des sources diverses. Or si l'on admet ce schéma de données qui se complètent mutuellement, on éprouve vite l'impression d'un système relativement complet et cohérent. Ainsi, alors que l'on avait cru identifier des lacunes importantes dans la loi des Saxons, et déploré notamment un silence total à propos du meurtre des hommes libres qui n'étaient pas 
membres de l'aristocratie, on s'est rendu compte récemment que ce cas juridique avait déjà été pris en compte auparavant dans les Capitulaires saxons qui, comme le montre la tradition manuscrite, étaient encore consultés en même temps que cette $1 \mathrm{li}^{50}$. Plus généralement, il a été rappelé que les lois coexistaient avec le droit oral, qu'elles invoquaient explicitement non seulement comme un souvenir du passé mais également comme une référence toujours valide ${ }^{51}$; ou encore avec d'autres champs normatifs tels que les serments, les règlements internes de telle ou telle corporation, etc ${ }^{52}$. Au final, la population du haut Moyen Âge pouvait éventuellement éprouver des difficultés à choisir entre des droits divers et parfois contradictoires, mais il n'y a pas lieu de s'inquiéter à propos de l'exhaustivité des normes auxquelles elle était soumise.

Il est vrai que ce dernier argument, comme les précédents, plaide certes pour la cohérence des lois mais n'en indique pas forcément la fonction : il est toujours possible de considérer, en effet, que cette association de textes complémentaires fut le fait d'un documentaliste particulièrement scrupuleux, et n'avait pas pour but d'informer un juge pour que celui-ci pût répondre à toutes les situations possibles. En revanche, les spécialistes ont accumulé récemment une série de données qui, elles, semblent aptes à faire pencher la balance du côté de la finalité pratique : il s'agit des cas dans lesquels le droit a été adapté à une situation nouvelle. On imagine mal, en effet, pourquoi un bibliothécaire, soucieux de conserver et de rassembler des informations issues du passé, aurait pris la peine de prendre en compte les modifications institutionnelles, les nouveaux rapports de domination, les nouveaux sentiments en matière d'ordre public de son temps : une telle mise à jour aurait été contre-productive dans la perspective d'une transmission d'information. En revanche, pour celui qui voulait fournir un outil de travail au juge, elle était non seulement utile, mais nécessaire pour permettre une prise de décision rapide. Or les exemples de ces adaptations, patiemment accumulés par les historiens du droit allemand dans les dernières années, sont légion. La loi romanoburgonde a ainsi modifié les choix qui avaient été opérés par sa principale source d'inspiration, la loi des Wisigoths, notamment à propos du droit de propriété et de vente es $^{53}$ ; pour sa part, la loi des Bavarois s'est également affranchie de ce même corpus ${ }^{54}$. On sait également que, tout au long du haut Moyen Âge, la promulgation de capitulaires vint compléter, amender et parfois contredire les lois édictées auparavant. Il existe certes des exceptions dans lesquelles le texte ne fut pas adapté à un nouveau contexte, produisant alors des normes difficiles à appliquer ${ }^{55}$; mais dans l'ensemble, on a bien l'impression de normes vivantes, en phase avec les besoins de la société qui les produisait. Pour conclure ce point, signalons que dans l'historiographie germanophone récente, aucune œuvre de synthèse n'a repris récemment la question de l'effectivité des lois pour réévaluer le modèle de H. Nehlsen; mais une série d'indices complémentaires, parmi lesquels cette adaptation permanente des lois, incite aujourd'hui la majorité des spécialistes à considérer, de facto, que les lois barbares furent destinées à une application judiciaire, et constituent une excellente source, de ce fait, pour l'histoire sociale de leur temps.

Tel est en effet le dernier axe de recherche développé par l'histoire du droit germanophone, et qui est peut-être le plus à même d'intéresser l'historien des évolutions sociales : passé la réflexion sur les conditions de production et sur la finalité des lois, que peuvent nous apporter ces dernières à propos des sociétés pour lesquelles elles furent conçues? Or sur ce sujet, le degré d'innovation de ces recherches fut variable: dans certains cas, les publications récentes se contentèrent de prolonger et de préciser les résultats des travaux antérieurs; dans d'autres, elles développèrent de nouveaux 
paradigmes; dans d'autres cas, enfin, elles résistèrent à l'innovation, et ce sera là l'occasion de présenter quelques desiderata de la recherche germanophone à propos de l'histoire du droit altimédiéval.

31 Un premier champ dans cet espace de recherche correspond aux catégories classiques de l'histoire sociale, politique et économique. En particulier, l'historiographie a sillonné le thème de la parenté et du genre, qui est bien représenté dans les lois barbares par les interdits d'endogamie, par les normes concernant les héritages, par les règles juridiques de domination masculine, ainsi que par la définition des groupes qui, lors des conflits, assistaient les parties en présence et constituaient ainsi le premier cercle social ${ }^{56}$. Par ailleurs, les recherches récentes ont prolongé les interrogations déjà séculaires en Allemagne à propos des structures hiérarchiques du haut Moyen Âge : le corpus juridique fournissait pour cela des indices à propos de la définition des catégories sociales, des relations entre ces dernières et de la mobilité sociale, ainsi que du traitement des esclaves ${ }^{57}$. Enfin, les structures économiques et commerciales ont également retenu l'attention des chercheurs ${ }^{58}$. Dans ces divers domaines, on vit fleurir ainsi des travaux utiles qui prolongeaient le fil des problématiques antérieures. On ne peut toutefois s'empêcher de noter deux restrictions qui affectent ces études: d'une part, aussi précieuses qu'elles soient, ce ne sont pas ces publications qui ont produit les résultats les plus novateurs dans l'histoire du droit altimédiéval. D'autre part, le volume global de ces publications reste modeste: on a l'impression que ces sujets, qui rassemblent pourtant l'essentiel des structures sociales du haut Moyen Âge, ont mobilisé moins d'énergie que la seule question technique et littéraire de l'origine des lois. Cette modestie des recherches est particulièrement frappante pour la question des relations hiérarchiques, qui avait drainé une part considérable de l'historiographie germanique dans les décennies précédentes. De toute évidence, ce n'est plus vers ces questions économiques et sociales que se sont tournées les forces vives de l'historiographie du droit dans les dernières années.

Un courant plus fertile fut celui qui analysait le traitement des coupables et la conception de l'ordre public qui en découlait. En ce domaine, les études récentes ont porté notamment sur l'attention que les lois accordaient à la psychologie du coupable; ces travaux permettaient ainsi un renouveau considérable de notre compréhension des politiques répressives. Avant eux, en effet, l'opinion reçue dans l'historiographie consistait à penser que les lois altimédiévales protégeaient l'ordre social avant la personne individuelle et qu'elles jugeaient donc essentiellement en fonction de la gravité du délit, des risques de violence que celui-ci impliquait, et du rang social du coupable et de la victime. Or des études systématiques ont pu montrer qu'en de nombreuses occurrences, on pouvait également déceler une prise en compte des motivations du coupable: non seulement le montant des compensations imposées montrait une distinction nette, par exemple, entre l'homicide, le meurtre et l'assassinat, mais la législation tenait compte également du lieu du crime, considérant qu'un meurtre dans la maison de la victime impliquait une préméditation ou, à tout le moins, un acharnement plus poussé que dans l'espace public ${ }^{59}$. Par-delà l'ordre social, le législateur altimédiéval prenait également en compte la conscience individuelle.

Mais cette attention apportée au traitement du coupable suscite actuellement des recherches plus novatrices encore, en particulier sous l'influence de Michel Foucault qui, auparavant, était singulièrement absent de l'histoire juridique allemande consacrée au haut Moyen Âge. C'est ainsi que Karl Ubl (Université de Tübingen) poursuit actuellement un projet de recherche financé par la Deutsche Forschungsgemeinschaft et portant sur la 
politique de la peine à l'époque carolingienne. L'auteur élargit ainsi un questionnement qu'il avait déjà abordé à propos de la question particulière de l'inceste, pour analyser les relations entre la peine, le processus de discipline sociale et la construction de l'autorité politique; il inclut dans cette recherche les éléments de l'imaginaire répressif, en particulier les peines évoquées dans les visions de l'au-delà, dans les œuvres poétiques ou dans les falsifications juridiques ${ }^{60}$. À l'aune de ce travail, on peut constater que dans l'historiographie récente, cette question a connu un itinéraire semblable à celui de l'ethnicité juridique que l'on décrivait plus haut : s'éloignant de travaux qui considéraient le délit comme une donnée a priori, à laquelle le législateur ne pouvait qu'apporter des solutions plus ou moins bonnes, le débat porte actuellement sur le caractère structurant de la norme pour la définition de la faute.

Un dernier thème, enfin, est appelé à un renouveau historiographique considérable même si, en l'occurrence, il faut davantage parler de lacunes à combler que de progrès effectués: il s'agit d'introduire les méthodes de l'ethnologie et les acquis de l'anthropologie dans le champ de l'histoire du droit germanophone ${ }^{61}$. Il est vrai que les premières tentatives en ce sens se sont heurtées à de fortes résistances de la part de l'historiographie du droit, soucieuse de défendre la spécificité de son corpus contre l'approche généraliste qu'elle attribuait à l'anthropologie. On fit valoir ainsi que les peuples de l'Europe altimédiévale n'étaient pas des sociétés exclusivement orales, et qu'une transposition des acquis de l'ethnologie serait donc inappropriée; plus généralement, on redoutait les anachronismes engendrés par un comparatisme mal maîtrisé, qui appliquerait des catégories trop générales au corpus des lois barbares. Les historiens du droit plaidèrent ainsi pour une prise en compte scrupuleuse des évolutions chronologiques, voire pour une étude au cas par cas ${ }^{62}$. Il est vrai que parmi eux, certains ont émis des avis plus nuancés ${ }^{63}$; mais même dans ce cas, les bénéfices attendus de l'ethnologie consistaient essentiellement à confirmer la fluidité des identités ethniques (et en ce domaine, l'interdisciplinarité a engendré un progrès incontestable pour l'étude des lois barbares) et l'existence de traditions orales dans les sociétés traditionnelles (un point qui, pour le coup, n'est pas très novateur dans le domaine altimédiéval). Pour d'autres thématiques développées par l'anthropologie historique internationale, en particulier pour les usages du corps, les rituels ou les symboles, la parcimonie de la bibliographie germanophone donne une idée du néant ${ }^{64}$.

Et pourtant, les lois barbares mériteraient mieux que cela, car elles constituent un gisement exceptionnel de pratiques sociales auxquelles les sciences sociales autres que l'anthropologie n'ont pas réussi à donner un sens; on se contentera ici de donner quelques exemples de ces énigmes non résolues. Parmi les gestes déroutants prescrits par ces lois, certains peuvent trouver une explication rationnelle rapidement : lorsque la loi des Bavarois impose de tirer les oreilles aux témoins commerciaux ${ }^{65}$, il s'agit sans doute, par-delà la simulation d'une sanction, de stimuler la mémoire du témoin pour que celuici, marqué par une douleur même symbolique, enregistre définitivement la transaction. On est déjà plus perplexe à propos des estimations précises, exprimées en valeur monétaire, que les lois barbares appliquent au corps humain, phalange par phalange ${ }^{66}$ : peut-être ce catalogue à la Prévert, à propos duquel on doute qu'il soit fonctionnel, étaitil destiné à fournir une théorie du corps humain qui permît de donner à celui-ci une place logique et hiérarchique dans l'ensemble du corps social? Surtout, l'historiographie germanophone reste frustrante à propos des gestes ou des mises en scène qui, dans les cérémonies de règlement d'un conflit, semblent peu fonctionnels a priori. Où trouver une 
explication pour cette clause de la loi des Bavarois qui obligeait le meurtrier d'un évêque à payer, comme réparation, le poids en or d'une tunique en plomb qui serait à la taille de cet évêque ${ }^{67}$ ? On serait tenté de considérer, mais la démonstration reste à faire, que ces modalités imposaient au coupable de simuler le don d'un équipement de protection au pontife qui, par sa fonction, ne pouvait en porter un: la nature même du dédommagement, et non pas seulement sa valeur financière, aurait ainsi contribué à symboliser la réparation et à faciliter le règlement du conflit. De même, quel sens donner à cette clause qui, dans la loi salique, impose aux débiteurs non solvables de jeter de la terre sur leurs proches avec leur main gauche, puis de parcourir le tour de leur maison vêtus d'une chemise ${ }^{68}$ ? Sans doute s'agit-il d'une mise en scène de la misère, destinée non seulement à humilier le coupable et ses proches (rappelons au passage que la main gauche est traditionnellement celle de l'impureté), mais également à marquer l'esprit des témoins, là encore, pour que ces derniers conservent la mémoire de cette déchéance. En ces diverses occurrences, on rencontre ainsi des mises en scène à forte composante visuelle, qui reconstituent, dans une construction théâtrale très poussée, le crime commis : le geste permet alors de produire du sens, s'adressant à la psychologie de la victime et justifiant, du même coup, le jugement. Il est ainsi possible de suggérer quelques pistes de recherche, mais au stade actuel, les Spielregeln qu'un Gerd Althoff a décryptées pour la sphère du pouvoir politique ${ }^{69}$ restent à découvrir dans le domaine du droit altimédiéval.

Cette étude touche à son terme. Par un choix quelque peu artificiel, elle s'est cantonnée aux seuls corpus législatifs et a ainsi laissé de côté les autres formes de normativité, alors que celles-ci ont probablement été appliquées en interaction constante avec la loi. Malgré ce choix conventionnel, notre panorama historiographique permet de prendre la mesure des grandes tendances récentes de l'historiographie germanophone à propos des lois barbares.

Avant tout, on a pu constater que certaines des questions majeures qui, depuis le milieu $\mathrm{du}$ XXe siècle, sillonnent l'historiographie allemande de manière lancinante ont également continué d'être appliquées au corpus qui nous occupe. Ainsi, le rôle joué par les Germains ou, plus généralement, par le facteur ethnique, a continué d'alimenter des discussions de grande ampleur. De même, les lois barbares voient toujours s'affronter les historiens qui envisagent, pour le haut Moyen Âge, un État fondé sur l'administration, et les théoriciens d'une société organisée plus spontanément sur la base du consensus : une discussion qui prolonge le dialogue, fondateur en Allemagne, entre Jürgen Habermas et Niklas Luhmann. Toutefois, ces débats de longue haleine n'ont pas empêché des profonds renouvellements : en particulier, les nouveaux courants de l'histoire du droit envisagent désormais une palette élargie des pratiques sociales qui permettaient de régler les conflits : par-delà la législation princière ou le droit canonique, une attention plus grande est désormais portée à d'autres sources juridiques, et également aux acteurs qui intervenaient avant ou après un éventuel passage au tribunal. D'autres nouveautés conceptuelles ont permis de considérer le droit altimédiéval non seulement comme un reflet d'une époque donnée, mais également comme un créateur de nouvelles structures sociales. Enfin, une dernière innovation de grande ampleur a permis à l'historiographie de prendre son parti de ce qu'elle considérait auparavant comme une maladresse des juristes du haut Moyen Âge, à savoir le silence des lois à propos de nombreuses situations conflictuelles. Certes, ces évolutions ne se sont pas faites sans heurts ni grincements de 
dents, mais elles ont néanmoins contribué à diversifier puissamment l'historiographie germanophone à ce sujet.

Comme conséquence de ce survol, est-il téméraire de proposer quelques lignes de synthèse à propos des spécificités des lois barbares, telles qu'elles apparaissent actuellement dans l'historiographie? Par rapport à la législation moderne, ce sont au moins deux éléments distinctifs qui caractérisent ce corpus législatif. En particulier, il semble désormais établi que les juristes du haut Moyen Âge avaient accès à plusieurs droits distincts et parfois divergents; même s'il n'est pas certain que ces divers textes étaient tous disponibles pour les juges, ni a fortiori pour les parties en présence, cette diversité offrait en tout cas un facteur de fluidité permettant de choisir entre plusieurs options selon les intérêts du moment. Par ailleurs, les lois barbares n'ont jamais cherché à envisager toutes les situations de la vie pratique: certes, il y eut des efforts incessants pour compléter le droit et pour l'adapter à de nouveaux publics, mais l'ampleur des situations non couvertes par la législation reste considérable. Ces lacunes peuvent faire du sens, si l'on se départit du schéma moderne qui considère la loi comme un Moloch destiné à avaler toutes les facettes de la vie sociale : au haut Moyen Âge, le juge pouvait se fier à un corpus contenu dans deux ou trois manuscrits, le reste étant soumis aux négociations entre les parties en présence. Par cette flexibilité permanente, les lois altimédiévale présentaient certes un caractère bien moins systématique que leurs homologues modernes; mais on peut supposer également qu'elles permettaient une plus grande attention à la situation sociale du moment. Au terme de cette révolution historiographique, les lois «barbares » paraissent décidément bien raffinées.

\section{NOTES}

1. Précisons que l'on se consacre ici à la période qui est postérieure au bilan historiographique proposé par Clausdieter Schott il y a trente ans: Clausdieter scHоTт, «Der Stand der LegesForschung » in : Frühmittelalterliche Studien, 13, 1979, p. 29-55

2. Philippe STURMEL, «L'école historique française du droit a-t-elle existé ? » in: Rechtsgeschichte. Zeitschrift des Max-Planck-Instituts für europäische Rechtgeschichte, 1, 2001, p. 90-121. Cet article est disponible en ligne: http://www.rg-rechtsge schichte.de/fileadmin/user_upload/PDF/rg01/ rg01_recherche_sturmel.pdf.

3. Zeitschrift der Savigny-Stiftung für Rechtsgeschichte. En réalité, cette revue en regroupe trois, puisqu'elle comporte une Germanistische Abteilung et une Romanistische Abteilung (depuis 1880), ainsi qu'une Kanonistische Abteilung (depuis 1911).

4. Avant 2001, il s'agissait de deux revues distinctes, Ius Commune - Zeitschrift für Europäische Rechtsgeschichte (depuis 1967) et Rechtshistorisches Journal (depuis 1982) ; depuis 2001, ces deux publications ont été regroupées dans un seul organe, Rechtsgeschichte Zeitschrift des Max-Planck-Instituts für europäische Rechtsgeschichte. Celui-ci est accessible en grande partie sur le site de l'institut : http://www.rg-rechtsgeschichte.de/.

5. Patrick WORMALD, The making of English law, Oxford et al . : Blackwell, 1999; Walter Pohl, «Leges Langobardorum» in: Heinrich BECK (dir.), Reallexikon der germanischen 
Altertumskunde, vol.18, 2ème édit., Berlin et al.: de Gruyter, 2001 (désormais : RGA), p. 208-213, ici p. 208.

6. Ruth SCHMIDT-WIEGAND, « Leges Alamannorum », RGA, p. 201-205, ici p. 201.

7. Isidore de Séville, Étymologies, V, 1, éd. WALLACE M. LINDSAY, OXFORD, 1911, vol. 1. Ce problème méthodologique est bien présenté, pour un autre corpus, par Harald Siems, «Lex Baiuvariorum » in: Adalbert ERLER et Ekkehard KAUFMANN (dir.), Handwörterbuch zur deutschen Rechtsgeschichte (désormais : HDR), vol. 2, Berlin : Schmidt, 1978, col. 1887-1901.

8. Karl UBL, «L'origine contestée de la loi salique. Une mise au point » in: Revue de l'Institut français d'histoire en Allemagne, 1, 2009, p. 208-234. Cette contribution est certes récente (et publiée dans une revue de bonne tenue), mais elle a déjà été contredite, durant la même décennie, par la plupart des spécialistes. Voir notamment Ruth SchmidtWiegand, « Lex Salica », RGA, p. 326-332, ici p. 326.

9. Voir à ce sujet H. Siems (H. SIEMS, « Lex Baiuvariorum », op. cit., ici col. 1891).

10. Id., « Lex Baiuvariorum », RGA, p. 305-315, ici p. 305.

11. Gerhard DILCHER, «Leges - Gentes - Regna » in: Id. et Eva-Marie DISTLER (dir.), Leges - Gentes Regna. Zur Rolle von germanischen Rechtsgewohnheiten und lateinischer Schrifttradition bei der Ausbildung der frühmittelalterlichen Rechtskultur, Berlin: Schmidt, 2006, p. 15-42, ici p. 18 (en particulier la note 9).

12. Voir notamment MaX DIESENBERGER et Walter POHL (dir.), Integration und Herrschaft. Ethnische Identitäten und soziale Organisation im Frühmittelalter, Wien: Österreichische Akademie der Wissenschaften, 2002.

13. Leges - Gentes - Regna..., op. cit.

14. Rappelons qu'il s'agit là d'un mode de réglementation extrajudiciaire des conflits, qui peut souvent se traduire par une vengeance privée.

15. Cette notion de Konsensgesetzgebung n'a été contestée nulle part dans l'historiographie allemande, du moins pour le haut Moyen Âge. Voir notamment Gerd KAMPERS, «Lex Burgundionum », RGA, p. 315-317, ou encore Ruth SCHMIDT-WIEGAND, « Lex Salica », op. cit., p. 326

16. Cette opinion de G. Dilcher n'est pas exceptionnelle: voir notamment Gabriele FRUSCIONE, «Zur Rolle von Ethnologie, Missionsgeschichte und angelsächsischen Rechtsquellen für die Erforschung germanischer Rechtsvorstellungen im Frühmittelalter - Eine Erwiderung " in: Zeitschrift der Savigny-Stiftung für Rechtsgeschichte. Germanistische Abteilung, 125, 2008, p. 399-410.

17. Voir notamment Herbert KALB, « Recht » in : Gert MELVILlE et Martial STAUB (dir.), Enzyklopädie des Mittelalters, Darmstadt: Primus, 2008, vol.1, p.189-193, ainsi que Theodor MAYER-MALY, «Römisches Vulgarrecht », HDR, vol. IV, 2008, col. 1132-1137, où l'on trouvera des références complémentaires

18. Alexander BECK, Itinera Iuris. Arbeiten zum Römischen Recht und seinem Fortleben, Bern : Stämpfli, 1980 ; plus récemment, voir la recension de Wolfgang Kaiser sur l'ouvrage de Charles M. RADDING et Antonio ciaralli, The Corpus Iuris Civilis in the Middle Ages. Manuscripts and Transmission from the Sixth Century to the Juristic Revival, Leiden : Brill (Brill's Studies in Intellectual History 147), 2007 : «Verkürzt und wiederaufgefüllt?» in: Rechtsgeschichte. Zeitschrift des Max-Planck-Instituts für europäische Rechtgeschichte, 11, 2007, p. 182-185.

19. C'est le cas en particulier dans l'espace lombard, où le droit romain, semble-t-il, fut curieusement moins exploité que dans les autres lois barbares. On sait pourtant qu'il restait pratiqué puisque Liutprand le proposa comme une option parmi d'autres pour celui qui voulait rédiger un diplôme (Walter POHL, «Leges Langobardorum », op. cit., p. 211); par ailleurs, l'Italie du nord était, sans surprise, l'espace dans lequel le droit romain était le mieux conservé (à ce sujet, voir les références fournies dans la note précédente). 
20. Les auteurs qui associent indissolublement faide et germanité sont nombreux: voir en particulier Patrick WORMALD, « Leges Anglo-Saxonum », RGA, p. 205-208, ou encore Ruth SCHMIDTWIEGAND, « Rechstvorstellungen bei den Franken und Alamannen vor 500 » in: Dieter GEUENICH (dir.), Die Franken und die Alemannen bis zur „Schlacht bei Zülpich“ (496/97), Berlin/ New York: de Gruyter (Ergänzungsbände zum Reallexikon der Germanischen Altertumskunde, 19), 1998, p. 545-557.

21. Ce qui suit remonte à Franz BEYERLE, «Die Malberg-Glossen der Lex Salica. Urspüngliche Randglossen. Stichworte zum jeweiligen Tatbestand» in: Zeitschrift der Savigny-Stiftung für Rechtsgeschichte, Germanistische Abteilung, 89, 1972, p. 1-32.

22. C'est cette conception qui motive actuellement l'organisation d'un programme de recherches intitulé Volkssprachige Wörter der Leges barbarorum («termes en langue vernaculaire dans les lois barbares »), élaboré par l'Institut für Frühmittelalterforschung de l'Université de Münster et financé par la Deutsche Forschungsgemeinschaft.

23. Voir notamment Elmar SEEBOLD, «Untersuchungen $\mathrm{zu}$ den malbergischen Glossen: Einführung " in: Beiträge zur Geschichte der deutschen Sprache und Literatur, 129, 1, 2007, p. 1-7. Précisons que la connaisance des langues germaniques anciennes n'est pas assez précise pour fournir un argument de datation: Kerstien Landwehr, «Über den volkssprachigen Wortschatz der Leges » in : Rudolf GROSSE (dir.), Bedeutungserfassung und Bedeutungsbeschreibung in historischen und dialektologischen Wörterbüchern, Stuttgart et al. : Hirzel, 1998, p. 73-76.

24. Agobard, Adversus legem Gundobardi, éd. Ernst DÜMMLER, Berlin : Weidmann (MGH Epistolae 5), 1899 , p. 158 suiv.

25. Voir en particulier G. DILCHER, op. cit., p. 27 suiv.

26. Martin SCHERMAIER, « Leges Romanae », RGA, p. 213-215.

27. Matthias SPRINGER, «Salier und salisches Recht - Beobachtungen zu den Wörtern Sali und Salicus» in : Die Franken Wegbereiter Europas : vor 1500 Jahren König Chlodwig und seine Erben, Mainz : P. von Zabern, 1996, vol. 1, p. 485-487. On serait d'ailleurs tenté de s'engager dans cette brèche pour interroger également la cohérence de la loi des Thuringiens, qualifiée de lex Anglorum et Werinorum hoc est Thuringorum dans les manuscrits. Ruth Schmidt-Wiegand, « Lex Thuringorum », $R G A$, suggère qu'il s'agit là d'un texte appliqué à une minorité d'Angles et de Warnes résidant auprès des Thuringiens; mais ne serait-il pas plus simple de considérer que, là encore, cette association d'ethnonymes ne répondait plus à la réalité sociale du IXe siècle durant lequel ce texte a été rédigé, mais à une de ces généalogies ethniques dont le haut Moyen Âge avait le secret, visant à accorder aux Thuringiens une ancienneté historique qu'ils n'avaient pas par référence à d'autres peuples mieux documentés ? Précisons toutefois qu'il ne s'agit là que d'une hypothèse, celle-ci n'ayant pas été démontrée ni même formulée, à notre connaissance, par l'historiographie allemande.

28. Walter POHL, « Leges Langobardorum », op. cit., p. 211.

29. Brigitte POHL-RESL, «Legal practice and ethnic identity in Lombard Italy » in: Walter Pohl et Helmut Reimitz (dir.), Strategies of Distinction : the construction of ethnic communities 300-800, Leiden : Brill, 1998, p. 205-220.

30. Gerhard DILCHER, « Langobardisches Recht », HDR, II, col. 1607-1618.

31. Harald SIEMS, « $\mathrm{Zu}$ Problemen der Bewertung frühmittelalterlicher Rechtstexte.» Zugleich eine Besprechung von R. Kottje, Zum Geltungsbereich der Leges Alamannorum » in : Zeitschrift der Savigny-Stiftung für Rechtsgeschichte. Germanistische Abteilung, 106 (1989), p. 291-305.

32. R. SCHMIDT-WIEGAND, « Leges Alamannorum », op. cit.

33. Signalons toutefois que pour cet espace géographique en particulier, l'historiographie allemande reste nettement plus favorable au système des lois personnelles, tandis que son homologue espagnole considère au contraire que la loi romano-wisigothique concernait 
l'ensemble de la population. Voir à ce sujet Detlef Liebs, "Lex Romana Visigothorum ", RGA, p. 323-326, ici p. 325, où l'on trouvera les références bibliographiques complémentaires

34. Harald SIEMS, Handel und Wucher im Spiegel frühmittelalterlicher Rechtsquellen, Hannover: Hahn (Monumenta Germaniae Historica, Schriften, 35), 1992, p. 270-276 et p. 612-614.

35. Hermann NEHLSEN, « Lex Burgundionum », HDR, II, col. 1901-1913.

36. Fritz STURM, « Persönlichkeitsprinzip », HDR, III, col. 1587-1597.

37. Hermann NEHLSEN, « Zur Aktualität und Effektivität germanischer Rechtsaufzeichnungen » in : Peter Classen (dir.), Recht und Schrift im Mittelalter, Sigmaringen: Thorbecke (Vorträge und Forschungen, 23), 1977, p. 449-502. Le texte est disponible en ligne: http://epub.ub.unimuenchen.de/9412/1/9412.pdf.

38. Une logique similaire a déjà été démontrée à propos des jugements de Dieu, qui sont d'ailleurs également mentionnés dans les lois barbares. Voir à ce sujet Thomas LIENHARD, «Ordalies et duels judiciaires au haut Moyen Âge (Ve-XIIe siècle) : en quoi croyait-on?» in : BullMHFA, 44, 2008, p. 211-231. Le texte est disponible en ligne : http://www.ifha.fr/allemagne/ Ordalies.pdf.

39. M. SCHERMAIER, « Leges Romanae », op. cit. On pourra consulter également Jon PEIDER ARQUINT, «Lex Romana Curiensis» in: Dictionnaire historique de la Suisse, vol. VII, Hauterive, 2007, disponible en ligne: http://hls-dhs-dss.ch/textes/f/F8943.php, qui fournit les références bibliographiques essentielles

40. Thomas LIENHARD, « Les usages de la loi » in : Marcelo Cândido da Silva, Bruno Dumézil et Sylvie Joye (dir.), Les lois barbares, à paraître, 2011.

41. H. SIEMS, "Lex Baiuvariorum» (comme plus haut, note 10); l'auteur fait référence notamment à une clause exigeant de tirer les oreilles aux témoins d'un accord commercial, et attestée par la diplomatique.

42. Brigitte POHL-RESL, « «Quod me legibus contanget auere ». Rechtsfähigkeit und Landbesitz langobardischer Frauen » in: Mitteilungen des Instituts für Österreichische Geschichtsforschung, 101, 1993, p. 201-227.

43. Pour ces questions manuscrites, un outil de première importance, même s'il ne se consacre pas prioritairement aux lois barbares, se trouve chez Hubert MORDEK, Bibliotheca capitularium regum Francorum manuscripta. Überlieferung und Traditionszusammenhang der fränkischen Herrschererlasse, München : Monumenta Germaniae Historica (Hilfsmittel, 15), 1995.

44. Parmi celles-ci, la plus connue est celle que composa Loup de Ferrières pour la loi salique (manuscrit de Modène, Archivio Capitolare O. I. 2). Voir à ce sujet Walter PoHL, Werkstätte der Erinnerung. Montecassino und die Gestaltung der langobardischen Vergangenheit, Wien et al.: Oldenbourg, 2001.

45. Ruth SCHMIDT-WIEGAND, « Lex Frisionum », RGA, p. 318-320, ici p. 319.

46. H. MORDEK, Bibliotheca capitulorum..., op. cit., p. 378 suiv.

47. H. SIEMS, « Lex Baiuvariorum » (comme plus haut, note 10), p. 311.

48. R SCHMIDT-WIEGAND, « Lex Frisionum », op. cit., p. 319

49. Friedrich Carl SAVIGNY, Geschichte des römischen Rechts im Mittelalter (1ère édit., 1815-1831), 2ème édit., Heidelberg/ Berlin : Mohr/ Trowitzsch, 1834, vol. II, p. 37-67 et vol. VII, p. 40-42.

50. Heiner LÜCK, « Lex Saxonum », RGA, p. 332-336, ici p. 334.

51. W. POHL, « Leges Langobardorum », op. cit., p. 209.

52. Ce point est utilement rappelé par H. KALB, « Recht », op. cit., p. 190-191.

53. H. SIEMS, Handel und Wucher ..., op. cit., p. 260-276 et p. 612-614. Voir également D. LIEBS, « Lex Romana Visigothorum », op. cit., p. 322. 
54. Isabella FASTRICH-SUTTY, Die Rezeption des westgotischen Rechts in der Lex Baiuvariorum : eine Studie zur Bearbeitung von Rechtstexten im frühen Mittelalter, Köln et al. : Heymanns, 2001.

55. Tel est le cas notamment de la clause qui, dans la loi romano-wisigothique, proscrivait le mariage entre Romains et Germains. Témoignant par sa formulation d'un profond sentiment de supériorité des Romains, cette norme, reprise du Code théodosien, fut sans doute difficile à appliquer à l'époque de la rédaction de la loi romano-wisigothique, alors que la péninsule Ibérique avait été conquise par les Wisigoths. Ce point a été relevé par D. LIEBS, «Lex Romana visigothorum ", op. cit., p. 324.

56. En plus de B. POHL-RESL, « «Quod me legibus contanget auere »... », op. cit., on pourra consulter également Raymund коттјЕ, «Eherechtlichen Bestimmungen der germanischen Volksrechte (5.-8. Jahrh. ) » in : Werner AFFELDT (dir.), Frauen in Spätantike und Frühmittelater. Lebensbedingungen - Lebensnormen - Lebensformen. Beiträge zu einer intern. Tagung am FB Geschichte der FU Berlin 18.-21-2-1987, Sigmaringen: Thorbecke, 1990, p. 211-220, ainsi que Friederike BAUER-GERLAND, Das Erbrecht der Lex Romana Burgundionum, Berlin : Duncker \& Humblot, 1995.

57. Gabriele VON OLBERG, Die Bezeichnungen für soziale Stände, Schichten und Gruppen in den Leges Barbarorum, Berlin/ New York: de Gruyter, 1991; Heung-Sik PARK, «Die Stände der Lex Saxonum » in : Concilium medii aevi, 2, 1999, p. 197-210.

58. H. SIEMS, Handel und Wucher ..., op. cit.

59. Voir en dernier lieu Dietmar WILLOWEIT, «Entdogmatisierung der mittelalterlichen Strafrechtsgeschichte» in: Rechtsgeschichte. Zeitschrift des Max-Planck-Instituts für europäische Rechtgeschichte, 14, 2009, p. 14-39.

60. Le projet est présenté sur le site Internet de l'Université de Tübingen: http:// www.mittelalter.uni-tuebingen.de/ ?q =forsch/forschung.htm\#strafen. Karl Ubl est également l'auteur de Inzestverbot und Gesetzgebung. Die Konstruktion eines Verbrechens (3001100), Berlin/ New York : de Gruyter, 2008.

61. Ce n'est d'ailleurs pas une spécificité germanique: dans le panorama historiographique qu'il propose à propos de l'anthropologie historique française appliquée au Moyen Âge, Jean-Claude Schmitt n'évoque pas le domaine juridique, ce qui est tout à fait représentatif pour l'actuelle situation des recherches en France. Jean-Claude schmiтT, "L'anthropologie historique de l'Occident médiéval. Un parcours" in: L'Atelier du Centre de recherches historiques, 07, 2010, disponible en ligne : http://acrh.revues.org/index1926.html.

62. On résume là le point de vue de Mathias scHMOECKEL, "Historische Anthropologie“ Anmerkungen eines Rechtshistorikers zu neueren Forschungen dieser Forschungsrichtung » in : Zeitschrift der Savigny-Stiftung für Rechtsgeschichte. Germanistische Abteilung, 119, 2002, p. 352-364. L'auteur s'oppose notamment à une publication particulière concernant l'usage de la torture à travers les âges, dans laquelle il relève un nombre important d'anachronismes. On serait tenté de lui rétorquer qu'un arbre ne peut se juger qu'à ses meilleurs fruits, et que l'anthropologie historique du droit médiéval, pour l'instant, est restée trop peu fertile pour offrir une base d'évaluation satisfaisante.

63. G. DILCHER, « Leges - Gentes - Regna », op. cit., notamment p. 18 suiv.

64. On pourra certes consulter Heinz DUCHHARDT et Gert MELVILLE (dir.), Im Spannungsfeld von Recht und Ritual. Soziale Kommunikation in Mittelalter und früher Neuzeit, Köln/ Weimar/ Wien : Böhlau (Norm und Struktur, 7), 1997 ; Ludger schwarTe et Christoph wUlf (dir.), Körper und Recht: anthropologische Dimensionen der Rechtsphilosophie, München : Fink, 2003 (notamment l'article de Christoph WULF, « Ritual und Recht. Performatives Handeln und mimetisches Wissen », p. 29-46) ; Reiner SCHULZE (dir.), Rechtssymbolik und Wertevermittlung, Berlin : Duncker \& Humblot, 2004 ; mais parmi ces trois publications, seule la première consacre quelques études au Moyen Âge, et aucune à la période altimédiévale. 
65. Voir plus haut, note XXX (40?).

66. Pactus Legis Salicae, 29, éd. Karl August Eckhardt, Hannover: Hahn (Monumenta Germaniae Historica Legum I, t. 4, 1), 1962, p. 112-117.

67. Lex Baiwariorum, I, 10, éd. Ernst von Schwind, Hannover: Hahn (Monumenta Germaniae Historica Legum I, t. V, 1), 1926, p. 281-282.

68. Pactus Legis Salicae (voir plus haut, note XXX (65 ?)), 58, p. 218.

69. Gerd ALTHOFF, Spielregeln der Politik im Mittelalter: Kommunikation in Frieden und Fehde, Darmstadt : Wissenschaftliche Buchgesellschaft, 1997.

\section{AUTEUR}

\section{THOMAS LIENHARD}

Thomas Lienhard est directeur de l'IFHA. 